\title{
A Fruit Quality Classification Algorithm Based on BP Neural Network and Computer Vision
}

\author{
Hequn Qiang ${ }^{1,2, a}$, Chunhua Qian ${ }^{1,3, b,{ }^{*}}$ and Yi Ren ${ }^{1, c}$ \\ ${ }^{1}$ Department of Computer Science, Suzhou Polytechnic Institute of Agriculture, Suzhou, China \\ ${ }^{2}$ Computer Science \& Technology, Soochow University, Suzhou, China \\ ${ }^{3}$ Department of Forestry Science, Nanjing Forestry University, Nanjing, China

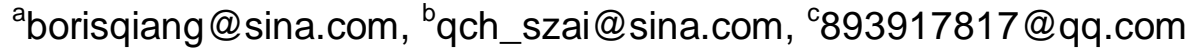

Keywords: BP Algorithm; Neural Network; Image Classification

\begin{abstract}
BP algorithm is a classical neural network algorithm. We analyzed the deficiency of traditional BP neural network algorithm, designed new S function and momentum method strategy, optimized the algorithm parameters. We use the new algorithm in the classification of orange images, take color and shape features as input value, the experimental results proved that our algorithm is faster and the classification accuracy rate reaches to $90 \%$.
\end{abstract}

\section{Introduction}

Fruit quality classification is very important to improve the income of farmers. At the present time, fruit quality classification mainly by hand, the whole process is waste of labour and inefficient. Therefore, research of automatic fruit quality classification technology based on computer image processing and neural network is significanct.

Since Rumelhart proposed BP algorithm in 1986, the neural networks developed fast and infiltrated into many subject[1]. The BP algorithm is widely used in computer image processing and pattern recognition.

In this paper, we used BP neural network algorithm to classify the orange images based on the color and shape features. Section 2 contain the analysis of traditional BP neural network algorithm. In section 3 , we designed the parameter optimization BP neural network algorithm. Section 4, we used the optimization algorithm to classify the orange images, gave the experimental results and conclusion.

\section{The Analysis of Traditional BP Neural Network Algorithm}

The structure of BP nerual network contains one input layer, one or more hidden layer and one output layer, each layer consists of a set of neurons, as shown in Fig.1.

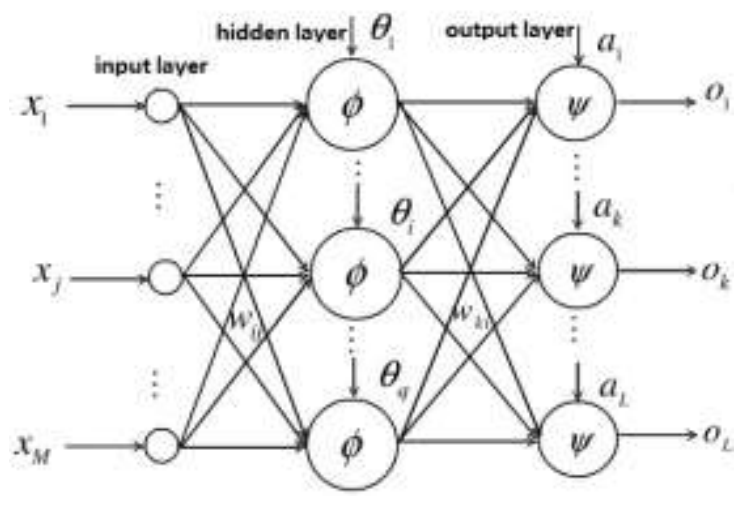

Fig. 1 the structure of BP nerual network 
The traditional BP neural network algorithm contains two mainly procedure: information forward transmission and error backward propagation. In the forward transmission procedure, information put into input layer, caculate by the hidden layer and the result come out from the output layer. The neurons' state in each layer just affect the neurons in the next layer. If the expected output is not obtained at the output layer, calculate the error variation values in output layer, then turn to the error backward propagation procedure which propagate the error variation values backward and modify the neuron weights in each layer to reach the expected output.

In BP neural network, neurons in the same layer are unconnect, neurons in different layers are forward connect. Take a three neural layer network as an example as shown in Fig. 1, number of input neurons is $\mathrm{M}$, number of hidden neurons is $\mathrm{L}, \mathrm{xp}_{1}, \mathrm{xp}_{\mathrm{j}} \ldots \mathrm{x}_{\mathrm{M}}$ is the input values, $O_{l}$ is the output values, $\mathrm{y}_{\mathrm{i}}$ is the output values of hidden neurons. $w_{i j}$ is the weights between the $\mathrm{i}$-th neuron in input layer and the $\mathrm{j}$-th neuron in hidden layer, $w_{k i}$ is the weights between the $\mathrm{k}$-th neuron in hidden layer and the $\mathrm{i}$-th neuron in output layer $(\mathrm{i}=1, \ldots, \mathrm{q}), \theta_{i}$ is the threshold of the $\mathrm{i}$-th neural in hidden layer, $a_{k}$ is the threshold of the k-th neural in output layer. $\phi(x)$ is the function of hidden layer and $\psi(x)$ is the function of output layer. We used the Sigmoid function called S function as well, as shown in Eq.1.

$$
f(x)=\frac{1}{1+e^{-x}}
$$

\section{The parameter optimization BP neural network algorithm}

In this section, we designed the process of fruit quality classification based on BP neural network algorithm as shown in Fig.2 and optimized the parameters of traditional algorithm.
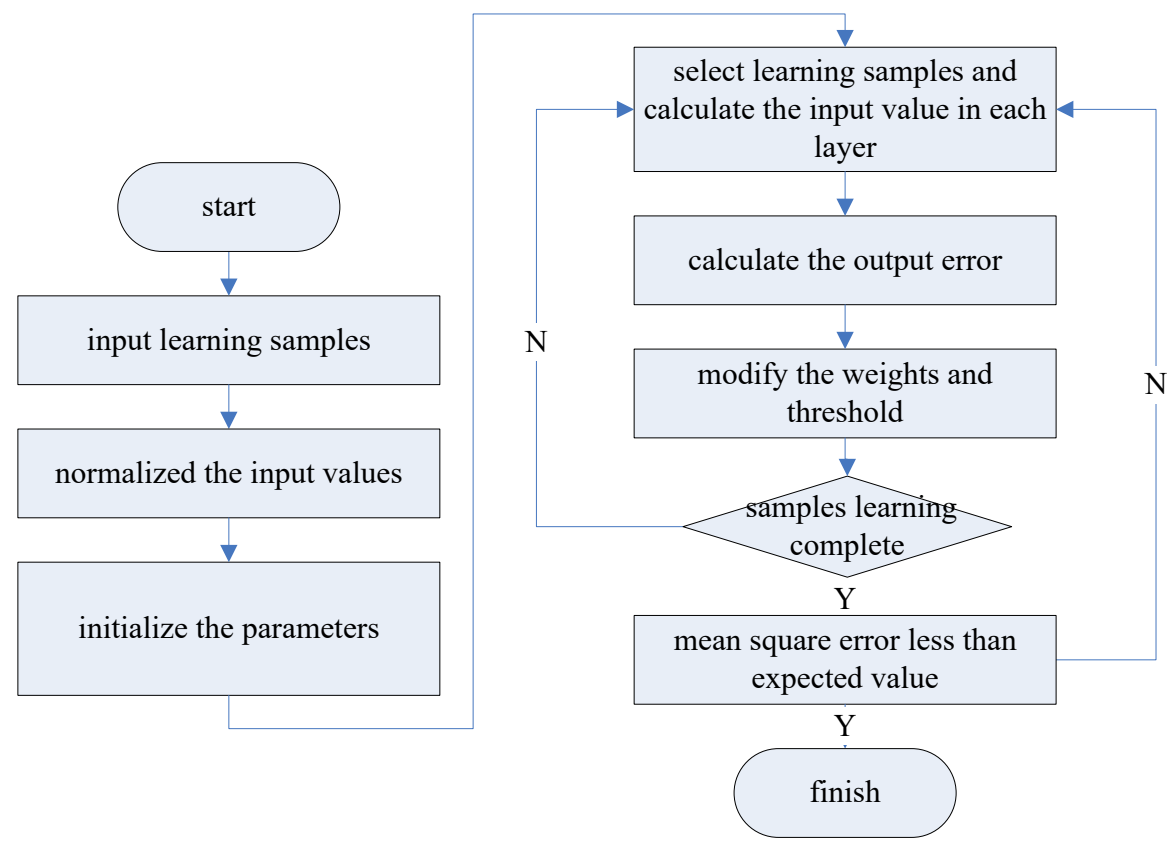

Fig. 2 the process of BP neural network algorithm

The color and shape feature values can be used as input values. The process contains six steps:

Step 1: Input samples for BP neural network learning. We select multi sets of different status parameter values as the study samples which can mostly reflect the various state.

Step 2: Initialize the parameters.Normalized the input values first, then initialize a set of parameters, include learning rate $\mu$, precision error $\varepsilon, \mathrm{S}$ function, the maximum number of training $\mathrm{k}$, the number of 
neurons in input layer $\mathrm{n}$, the number of neurons in hidden layer $\mathrm{k}$, the number of neurons in output layer $\mathrm{m}$, threshold of hidden layer $\theta_{k}$, threshold of output layer $\theta_{m}$.

Step 3: caculate input and output value in each layer according to Eq.2 and Eq.3

$$
\begin{aligned}
& \text { net }_{k}=\sum_{i=1}^{q} w_{k i} y_{i}+a_{k}=\sum_{i=1}^{q} w_{k i} \phi\left(\sum_{j=1}^{M} w_{i j} x_{j}+\theta_{i}\right)+a_{k} \\
& o_{k}=\psi\left(\text { net }_{k}\right)=\psi\left(\sum_{i=1}^{q} w_{k i} y_{i}+a_{k}\right)=\psi\left(\sum_{i=1}^{q} w_{k i} \phi\left(\sum_{j=1}^{M} w_{i j} x_{j}+\theta_{i}\right)+a_{k}\right)
\end{aligned}
$$

Step 4: caculate output error according to Eq.4 , if $\mathrm{E}(\mathrm{t})<\varepsilon$, goto Step 6, otherwise goto Step 5;

$$
E=\frac{1}{2} \sum_{P=1}^{P} \sum_{k=1}^{L}\left(T_{k}^{p}-O_{k}^{p}\right)^{2}
$$

Step 5: modify the weights according to Eq.5;

$$
w_{i j}(t+1)=w_{i j}(t)-\eta \Delta w_{i j}(t)
$$

Step 6: finish the process.

We use color and shape feature to research orange quality classification, it requires fast convergence and high accuracy, so we optimize the parameters of the traditional BP neural network algorithm.

(1) Selecting initial weights: it is very important for the selection of the initial value of the nonlinear system, it directly affects the learning convergence and the training time. We select random number between -1 and 1 .

(2) The learning rate: Weight variation in each cycle training is determined by the learning rate. High learning rate can lead to system instability and small learning rates lead to longer training time. Therefore, we set an automatically adjust learning rate.

(3) The additional momentum method: this method can avoid algorithm falling into local minimum. Weights and threshold adjustment with additional momentum factor as shown in Eq.6 and Eq.7

$$
\begin{aligned}
& \Delta w_{i j}(k+1)=(1-m c) \eta \delta_{i} p_{j}+m c \Delta w_{i j}(k) \\
& \Delta b_{i}(k+1)=(1-m c) \eta \delta_{i}+m c \Delta w_{i j}(k)
\end{aligned}
$$

Where $\mathrm{k}$ is maximum number of training, $\mathrm{mc}$ is momentum factor.

The value of $\mathrm{mc}$ is determined by Eq. 8

$$
m c=\left\{\begin{array}{cc}
0 & E(k)>E(k-1) * 1.04 \\
0.95 & E(k)>E(k-1) \\
m c & \text { others }
\end{array} \quad, \quad E(k) \text { is sum of squared errorin step } k\right\}
$$

(4) modify the S function. In traditional BP neural network algorithm, S function is shown as Eq.1. it is fixed and lower the convergence speed. So we modify the function shown as Eq.9

$$
f(x, s, \sigma)=\frac{1}{1+e^{-s(x+\sigma)}}
$$

Compare to the traditinal $\mathrm{S}$ function, we add an adjustable offset parameter $\sigma$ in it. Slope $\mathrm{S}$ and offset $\sigma$ are modified with the error signal in the error backward propagation procedure, they speed up the convergence obviously and improve the adaptive ability of neurons.

(5) the number of hidden layer: So far there is not a unified standard for the choice of it. We use Eq. 10 to caculate the number of hidden layer 


$$
m=\sqrt{n+l}+a
$$

Where $\mathrm{m}$ is the number of hidden layer, $\mathrm{n}$ is the number of input layer, 1 is the number of output layer, $\mathrm{a}$ is constant between 0 and 10 .

\section{Experimental Results and Conclusion}

We do two types of experiment with color feature and shape feature respectively under Matlab.

In color feature classification experiment, the number of input layer $n=8$ which represent eight hue value, output layer number $\mathrm{l}=4$ which represent four classes of oranges. the number of hidden layer $\mathrm{m}=8$, learning rate $\mu=0.3$, precision error $\varepsilon=0.01$, weight $\mathrm{w}=0.3$ maximum number of training $\mathrm{k}=5000$, after 860 times training, the precision error $\varepsilon<0.01$, the classification accuracy rate reach to $95 \%$.

In shape feature classification experiment, the number of input layer $n=13$ which represent thirteen Fourier descriptor operator, output layer number $1=4$ which represent four classes of oranges. the number of hidden layer $\mathrm{m}=8$, learning rate $\mu=0.3$, precision error $\varepsilon=0.01$, weight ${ }_{\mathrm{w}}=0.3$ maximum number of training $\mathrm{k}=6000$, after 1030 times training, the precision error $\varepsilon<0.01$, the classification accuracy rate reach to $90 \%$, the error curve shown as Fig.3.

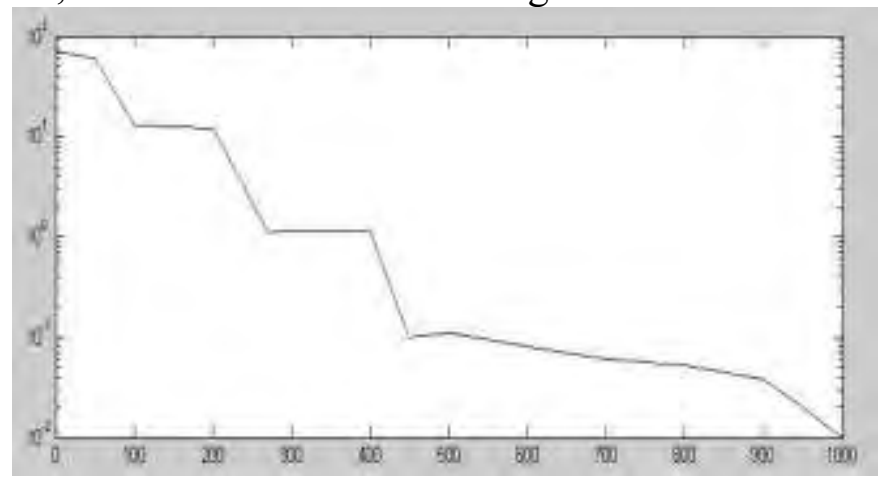

Fig. 3 error curve of the training with shape feature

In this paper, we optimized the parameters of the traditional BP neural network algorithm and used it to classify the orange images with color and shape features. The experimental results proved that the algorithm we optimized is effective and accurate.

\section{Acknowledgement}

This paper was financially supported by the Scientific research fund for young teachers of Suzhou Polytechnic Institute of Agriculture(PPN201511) and The project of Suzhou Agriculture Commission -mordern agricultural parks(jd201711).

\section{References}

[1] David E. Rumelhart, Geoffrey E. Hinton, Ronald J. Willams. Learning representations by back-propagating errors[J]. Nature 1986, Vol.323, 533-536

[2] I Sa,Z Ge,F Dayoub,B Upcroft. DeepFruits: A Fruit Detection System Using Deep Neural Networks[J]. Sensors. 2016, Vol16 (8) :1222

[3] EO Olaniyi, OK Oyedotun, K Adnan. Intelligent Grading System for Banana Fruit Using Neural Network[J]. Arbitration Journal of Food Process Engineering. 2017 , 40 (1)

[4] JDJ Rubio. A method with neural networks for the classification of fruits and vegetables[J]. Soft Computing. 2016,21(23):1-14

[5] Y Zhang, L Wu. Classification of Fruits Using Computer Vision and a Multiclass Support Vector Machine[J]. Sensors,2012,12 (9) :12489-12505

[6] S Bargoti, JP Underwood. Image Segmentation for Fruit Detection and Yield Estimation in Apple Orchards[J]. Journal of Field Robotics. 2017 , 34 (6) 